\section{Inner Lumen Tracheostomy Tube Capable of Continuous Monitoring of Cuff Pressure}

\author{
Vahid Saadatmand ${ }^{1 * \odot}$, Hassan Khaledi Sardashti', \\ Mahmoud Reza Peyravi², Mohammad Hasan Amirsalari
}

\begin{abstract}
Tracheostomy is one of the most important airway management procedures in patients with respiratory problems. This procedure might be the only option for patients with airway and respiratory problems, who are not able to have an appropriate ventilation and oxygenation. For this purpose, tracheostomy tubes are used with different applications. One of these types of tubes is the cuffed tracheostomy tube, used for fixation, positive pressure ventilation and aspiration prevention. Nevertheless, there are two common problems with using these regular tracheostomy tubes. First, these tracheostomy tubes do not have a unique cuff pressure monitoring system. Although there are portable monitoring devices for such purpose in intensive care units (ICU), they are shared among several patients, increasing the risk of infection transmission. Second, due to the presence of the patient's thick secretions, the tracheostomy tube becomes obstructed and inefficient. The pathway does not get open even through suction and thus the tracheostomy tube must be replaced. In order to resolve these problems, the inner lumen tracheostomy tube capable of continuous monitoring of cuff pressure has been invented with two techniques, which set it apart from other related tools. The invented optimized tracheostomy tube has been developed with different techniques through creating a disposable monometer system attached to it as well as a removable inner lumen embedded in it.
\end{abstract}

Citation: Saadatmand V, Khaledi Sardashti H, Peyravi MR, Amirsalari MH. Inner Lumen Tracheostomy Tube Capable of Continuous Monitoring of Cuff Pressure. J Biomed Phys Eng. 2021;11(6):757-760. doi: 10.31661/jbpe.v0i0.2012-1247.

\section{Keywords}

Airway Management; Intubation; Tracheostomy

\section{Introduction}

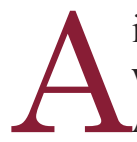
irway management is the high priority in the treatment of patients with respiratory problems, and the tracheostomy is among common Airway management procedures. In this procedure, a hole is created in the anterior wall of the trachea to establish airway. This technique is often temporary and reversible. Tracheostomy has been used for more than 2000 years and developed and progressed by now [1] that this procedure changed since 1909. The incision in the tracheostomy procedure should be made below the second tracheal ring to prevent adverse events under the second layer of the tracheal ring to prevent adverse events to the extent possible [1, $2]$. In the past, the most common indication of tracheostomy was obstruction of the upper airway caused by infectious diseases. However, infectious causes are no longer the most common indications of tracheostomy due to vaccination and the use of antibiotics. In most studies, long-term intubation of more than 13 days has been the most common indication of tracheostomy [3].

Despite its many benefits, tracheostomy procedure has dangerous side effects, which can be highly dangerous and even irreversible if they are neglected. One of the most important side effects is tracheal mucosal injury due to excessive distension of the tube cuff (more than $30 \mathrm{~cm} \mathrm{H}_{2} \mathrm{O}$ ), putting
${ }^{1} \mathrm{PhD}$ Candidate, Department of Health in Emergencies and Disasters. Shiraz University of Medical Sciences,

Shiraz, Iran

${ }^{2} \mathrm{MD}$, PhD, Department of Health in Emergen-

cies and Disasters, School of Management and Medical Informat-

ics, Shiraz University

of Medical Sciences,

Shiraz, Iran

${ }^{3} \mathrm{BSc}, \mathrm{EMT}$, Emergency Medical Service of Jahrom University of Medical Sciences, Jahrom, Iran

*Corresponding author: Vahid Saadatmand Department of Health in Emergencies and Disasters. Shiraz University of Medical Sciences,

Shiraz, Iran

E-mail: vahid.saadat60@ gmail.com

Received: 16 December 2020 Accepted: 18 January 2021 
pressure on the capillaries of the tracheal wall, which normally has a pressure of $22 \mathrm{mmHg}$, and thus results in the occurrence of such side effects as erosion, inflammation and softening of cartilage rings, tracheal distension, bleeding, infection and stenosis of the trachea. However, insufficient distension of the tracheostomy tube cuff (less than $18 \mathrm{~cm}$ of $\mathrm{H}_{2} \mathrm{O}$ ) results in aspiration of the secretions in the upper airways. To prevent the occurrence of such complications, the pressure inside the cuff should be recorded at appropriate time intervals and minimum pressure should be provided with an appropriate volume [1-3]. Currently, to determine whether the tube cuff is filled, the outer cuff balloon is touched and estimation is used, which are not reliable and accurate at all, even if done by skilled and professional individuals [4].

A problem with patients that have long-term intubation and tracheostomy is pulmonary infections and secretions with a high viscosity, which narrow and obstruct airways and ultimately disrupt oxygen exchange. Suction is used to resolve this issue. However, in the long term, the obstruction reaches a level that severely narrows the tube's interior caliber, disrupting an appropriate airflow through the tube. Thus, the tube must be changed. However, this change and replacement will have such side effects as increased risk of infection, bleeding and tracheal stimulation $[1,5]$.

Considering the importance of cuff monitoring of tracheostomy tubes (in patients with mechanical ventilation) in ICUs and prevention of frequent and early replacement of tracheostomy tube following obstruction (due to secretions with a high viscosity), this optimized device was invented to resolve the above said issues, reduce nosocomial infections, prevent further traumas following re-intubation and save time.

\section{Technical Presentation}

The technique for constructing this devise is as follows: We develop the tracheostomy tube made of polyethylene based on the trachea's anatomical structure in a curved shape and with a length of $10 \mathrm{~cm}$. Then at the ending $3 \mathrm{~cm}$ of the tube, the air-filled cuff is embedded. Inside the cuff, we remove a capillary tube made of polyethylene with a diameter of $1 \mathrm{~mm}$ from the tube's wall in such a way that the tube's extension passes through the tract embedded in the corner of the tracheostomy tube's butterfly-shape wing. This capillary tube ends up in three pathways. The three pathways include:

A) A pathway attached to a one-way vent for air to come in and end up in an air balloon and used to take the amount of air necessary for filling the tracheostomy tube cuff and adjusting the cuff pressure (filling and emptying the cuff).

B) A pathway ending up in a manometer, which is sensitive to air pressure and the numbers written on it are based on centimeter of $\mathrm{H}_{2} \mathrm{O}$. The amount of pressure recorded on it represents the pressure inside the cuff.

C) A pathway turning towards the tracheostomy tube cuff.

First, the entrance of the tube is made with different diameters compared to other parts of the tube so that it would be compatible with the bag valve mask's opening and the ventilator tube. In the first $3 \mathrm{~cm}$ of the tube, there are two fittings with the shape of open wings of a butterfly, which aim at fixing the tube through two fabric tapes. In the inner caliber of the tracheostomy tube, a tube with a diameter of less than $0.5 \mathrm{~mm}$ and equal in size to the tracheostomy tube made of polyethylene is embedded, covering the whole inner diameter of the tube and is considered as the inner layer. This layer is able to move and exit the opening of the tracheostomy tube. At the beginning of its entrance into the tube, there is a layer of it extended outwards so that it can be used as a handle to discharge it during filling with secretions and the tube's inner caliber diameter is narrowed.

The parts of this device are descried below and shown in Figure 1:

1. One-way vent for entrance into the circuit: This vent has been embedded for one-way entrance of air into the cuff manometer circuit, made up of a plastic external balloon and a oneway spiral entrance.

2. Three-way valve: This three-way valve accounts for the fitting between the one-way vent, manometer and tube cuff.

3. Manometer: This is used to determine the amount of pressure inside the tube cuff in centimeters of $\mathrm{H}_{2} \mathrm{O}$.

4. Air-to-cuff transfer tube: This includes a capillary tube with a diameter of around $1 \mathrm{~mm}$ to transfer air from the one-way vent to the cuff.

5. Connection to the bag valve mask: This 


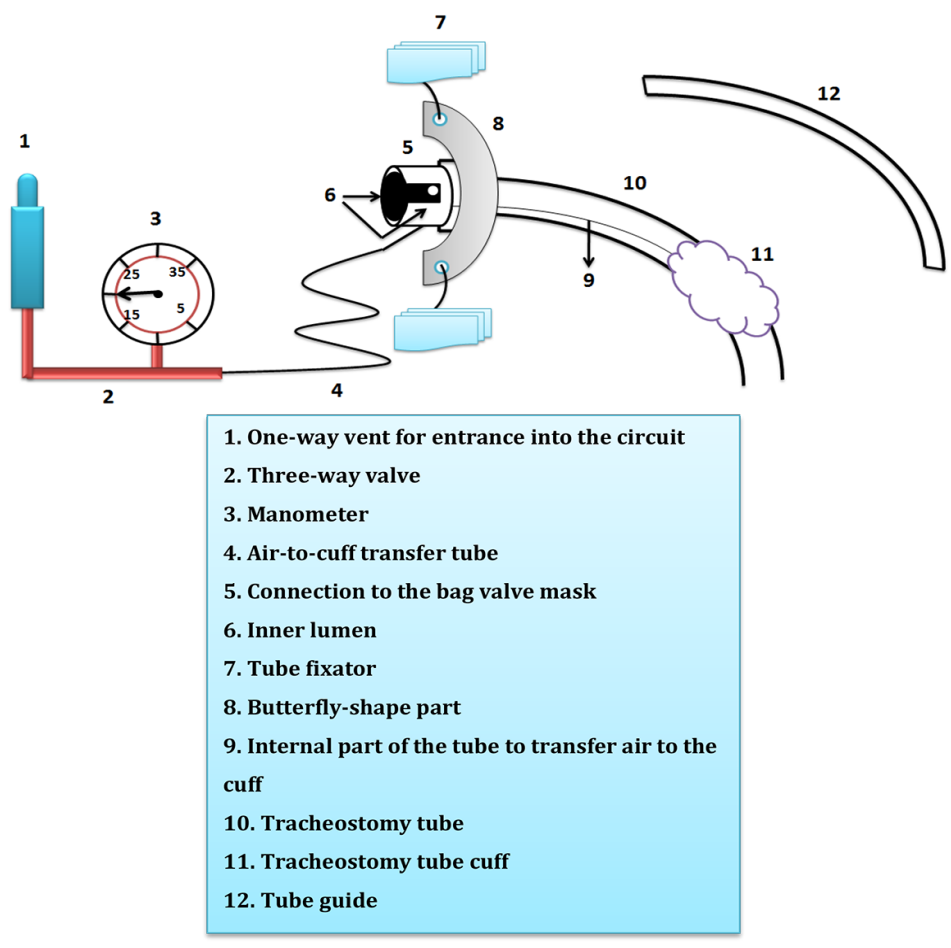

Figure 1: Schematic inner lumen tracheostomy tube with the ability of continuous cuff pressure monitoring.

is a fitting connected to the beginning of the tracheostomy tube, designed for adjustment to the tip of the bag valve mask or the ventilator's tube.

6. Inner lumen: This part is made of polyethylene and has an internal wall that can be removed from the inside of the tube. Its external part is folded on the tube as its handle.

7. Tube fixator: This includes a fabric part, fixing the tube on the embedded neck.

8. Butterfly-shape part: This is the tracheostomy tube's scalp part, located at a distance of around $3 \mathrm{~cm}$ of the beginning of the tube and designed to fixate the tube.

9. Internal part of the tube to transfer air to the cuff: This is part of the capillary tube that passes through the scalp within the internal wall of the tracheostomy tube and is finally connected to the cuff.

10. Tracheostomy tube: This is made of polyethylene and its shape has been designed based on the anatomical structure.

11. Cuff: This is an air-filled balloon in the distal part of the tracheostomy tube, causing the tube to be fixed to the trachea's internal wall from inside.

12. Tube guide: This is flexible and made of aluminum and also used to facilitate the intuba- tion process.

\section{Discussion}

Tracheostomy tubes have been designed with different techniques and capabilities. Norbert Neubauer (2015) invented a tracheostomy cannula with a large cuff and low pressure as well as the capability to suction using vacuum tools attached to the tube [6]. Bastian Ramdohr (2015) invented a tracheostomy tube whose major characteristic was a high level of stability when moving the patients [7]. Ti-Li Chang (2013) invented a tracheostomy tube with an internal tube in which a replaceable internal layer with the main cannula of tracheostomy tube is attached, which has an air passage tract and a pressure cuff. Meanwhile, it can be removed from the external layer when necessary [8]. Normal C. Crandall (1982) invented a tracheostomy tube with an internal disposable cannula. The internal layer was made of polyvinyl chloride and was able to be removed from the outer tube [9].

Our innovative device has two major differences from other common tracheostomy tubes. Firstly, this device is equipped with a cuff pressure monitoring part that is attached to it and has the ability to continuously monitor the cuff pressure, 
while in other devices, the cuff pressure monitoring is done through portable and alternating devices. Secondly, the inner lumen is designed in such a way that it comes out of the main tube easily during replacement and there is no need for re-intubation and related problems.

The most important benefits of this device are related to its design techniques. This device has a manometer, continuously monitoring the cuff pressure and preventing the risk of aspiration and structural damage to the tracheal wall. Also, the presence of a disposable and replaceable inner lumen reduces the risks of re-intubation, including trauma and infection. Another advantage of this device is to be cheap and available. Although this device has unique benefits, perhaps the most important limitation is that the user of this device must be a trained and educated person, especially in situations where this device is used to care for the patients at home.

\section{Conclusion}

The inner lumen tracheostomy tube capable of continuous monitoring of cuff pressure has been designed with two techniques, performed by attaching a manometer to continuously monitor the cuff pressure and installing an interchangeable inner lumen in the main tube to prevent early reintubation. In advanced airway management, this device can reduce many problems of common tracheostomy tubes such as aspiration, tracheal damage, trauma and infection.

\section{Acknowledgment}

We sincerely appreciate the Department of Research and Center for Development and Technology of Jahrom University of Medical Sciences and the Department of Research of Shiraz University of Medical Sciences for helping us in this study.

\section{Authors' Contribution}

Saadatmand V and Amirsalari $\mathrm{H}$ conceived the idea. Introduction of the paper was written by Peyravi MR and Khaledi H. Saadatmand V and Amirsalari $\mathrm{H}$ gather the images and the related literature and also help with writing of the related works. The method implementation was carried out by Saadatmand V. conclusion and discussion was carried out by Saadatmand V, Peyravi MR.
The research work was proofread and supervised by Saadatmand V. All the authors read, modified, and approved the final version of the manuscript.

\section{Ethical Approval}

This project is related to the invention of a medical device and has been registered in the intellectual property and document registration office of Iran under the number 1395/07/10-90019.

\section{Funding}

Part of the costs related to this project was provided by the growth and technology center of jahrom university of medical sciences and the other part was provided personally.

\section{Conflict of Interest}

None

\section{References}

1. Dawson D. Essential principles: tracheostomy care in the adult patient. Nurs Crit Care. 2014;19(2):63-72. doi: 10.1111/nicc.12076. PubMed PMID: 24529106.

2. Kim DM, Shin MJ, Kim SD, et al. What is the Adequate Cuff Volume for Tracheostomy Tube? A Pilot Cadaver Study. Ann Rehabi Med. 2020;44(5):402-8. doi: 10.5535/arm.19210. PubMed PMID: 32986943. PubMed PMCID: PMC7655230.

3. Cheung NH, Napolitano LM. Tracheostomy: Epidemiology, Indications, Timing, Technique, and Outcomes. Respir Care. 2014;59(6):895-919. doi: 10.4187/respcare.02971. PubMed PMID: 24891198.

4. Morris LG, Zoumalan RA, Roccaforte JD, Amin MR. Monitoring tracheal tube cuff pressures in the intensive care unit: a comparison of digital palpation and manometry. Ann Otol Rhinol Laryngol. 2007;116(9):639-42. doi: 10.1177/000348940711600902. PubMed PMID: 17926583.

5. Withe AC, Kher S, O'connor HH. When to Change a Tracheostomy Tube. Respiratory Care. 2010;55(8):1069-75. PubMed. PMID: 20667154.

6. Neubauer N. Tracheostomy cannula with low pressure cuff and suction device. Google patents/ DE202010001611U1; 2015. Available from: https:// patents.google.com/patent/DE202010001611U1/en.

7. Ramdohr B, Trger P, Klimenta K. Tracheal cannula inner tube. Google patents/W02015131867A1; 2015. Available from: https://patents.google.com/ patent/W02015131867A1/de.

8. Chang TL, Chiu Sy. Inner Type Tracheostomy Tube. Google patents/ US20130269690A1; 2013. Available from: https://patents.google.com/patent/ US20130269690A1/en.

9. Crandall NC, French RC. Tracheostomy tube with disposable inner cannula. Google patents/ US4315505A; 1982. Available from: https://patents. google.com/patent/US4315505A/en. 